\title{
Neuropsychological function in patients with a single gene mutation associated with autosomal dominant nocturnal frontal lobe epilepsy
}

\author{
Amanda G. Wood ${ }^{\mathrm{a}, \mathrm{b}, *}$, Michael M. Saling ${ }^{\mathrm{c}, \mathrm{d}}$, Marco Fedi ${ }^{\mathrm{e}}$, Samuel F. Berkovic ${ }^{\mathrm{e}}$, Ingrid E. Scheffer ${ }^{\mathrm{e}, \mathrm{f}}$, \\ Christopher Benjamin ${ }^{a}$, David C. Reutens ${ }^{\text {a,g }}$ \\ a Department of Medicine, Southern Clinical School, Monash University, Melbourne, Australia \\ ${ }^{\mathrm{b}}$ Critical Care and Neurosciences, Murdoch Childrens Research Institute, Royal Children's Hospital, Parkville, Australia \\ ${ }^{\mathrm{c}}$ Department of Psychology, University of Melbourne, Melbourne, Australia \\ ${ }^{\mathrm{d}}$ Neuropsychology, Austin Health, Melbourne, Australia \\ e Epilepsy Research Centre, Department of Medicine, University of Melbourne, Australia \\ ${ }^{\mathrm{f}}$ Department of Paediatrics, University of Melbourne, Royal Children's Hospital, Australia \\ ${ }^{\mathrm{g}}$ Centre for Advanced Imaging, University of Queensland, Australia
}

\section{A R T I C L E I N F O}

\section{Article history:}

Received 9 December 2009

Revised 29 January 2010

Accepted 29 January 2010

Available online 26 February 2010

\section{Keywords:}

Cognition

Epilepsy

Genetic

Nicotinic acetylcholine receptor

Autosomal dominant nocturnal frontal lobe epilepsy

\begin{abstract}
A B S T R A C T
Autosomal dominant nocturnal frontal lobe epilepsy (ADNFLE) is a nonlesional condition associated with mutation of the gene coding for the $\alpha 4$ nicotinic acetylcholine receptor ( $\mathrm{AAChR}$ ). The nAChR modulates aspects of memory and attention. We examined the neuropsychological phenotype of ADNFLE, with a particular emphasis on understanding the impact on frontal lobe functions. We used standard clinical tests as well as focused measures of frontal lobe function in a well-defined group of patients with ADNFLE. Their performance was compared with that of a group of age-, sex-, and education-matched control participants. Patients with ADNFLE showed impairments on tasks requiring cognitive flexibility against a background of well-preserved intellectual abilities. In accord with existing research, verbal memory impairments were identified in the patient group; the level of impairment on these tasks correlated with disease-related factors. In our study of ADNFLE associated with one mutation, cognitive flexibility appears to be the core cognitive deficit.
\end{abstract}

Crown Copyright @ 2010 Published by Elsevier Inc. All rights reserved.

\section{Introduction}

Autosomal dominant nocturnal frontal lobe epilepsy (ADNFLE) was the first epilepsy syndrome for which a genetic basis was discovered, and several nicotinic receptor subunit gene mutations have now been reported [ $1-3]$. Neuronal nicotinic acetylcholine receptors (nAChRs) are heteropentamers of $\alpha(2-10)$ and $\beta(2-4)$ subunits and are widespread throughout the brain. Initial human and animal evidence of a role for these receptors in fundamental and higher-level aspects of cognition has been reported, but a definitive view is yet to emerge. Administration of nicotine to abstinent smokers improves general alertness, attention, and vigilance [4,5]. Nicotine improves performance on vigilance tasks [6,7], inhibition of prepotent responses [8], and verbal learning [9] in nonsmokers. Effects such as these, however, are thought to reflect an influence on general alertness, rather than the primary influence of specific cognitive domains $[10,11]$. Administration of nicotine or nicotinic drugs might also facilitate attention and memory in patients with dementia of the Alzheimer type [12]. The predominant effect of nAChR ligands in

\footnotetext{
* Corresponding author. Address: MRI Department, Level 2, Royal Children's Hospital, Flemington Road, Parkville 3052, Australia. Fax: +61 393454325

E-mail address: amanda.wood@mcri.edu.au (A.G. Wood).
}

experimental animals appears to be on memory processing $[6,13]$, and the $\alpha 7$ and $\alpha 4 \beta 2$ receptors have been implicated in mediating these effects [5].

In the current study we examined individuals with a missense mutation, S248F, of the CHRNA4 gene that encodes the $a 4$ subunit of the nAChR $[3,14]$. Patients with this mutation have ADNFLE, a distinctive syndrome associated with mutations of three acetylcholine receptor subunit genes, CHRNA4, CHRNB2, CHRNA2, in different families [15]. The majority of mutations have been found in CHRNA4. Patients with ADNFLE experience clusters of nocturnal seizures arising from the frontal lobes [14], and reduced right prefrontal receptor density and hypometabolism are observed on PET [16]. The frontal lobe aspects of the disorder raise the possibility that associated cognitive dysfunction may be observed. Research on a pair of monozygotic twins with a mutation of the $\beta 2 \mathrm{nAChR}$ found impaired verbal memory abilities [17]. Recent data on a group of 11 patients with known nAChR subunit mutations (three CHRNA4 and one CHRNB2 mutation) [16] showed impaired memory and executive function as well as impaired intellectual abilities in 5 patients.

The aim of the current study was to characterize neurocognitive function in patients with ADNFLE caused by the same CHRNA4 mutation, tapping a range of functions that are heavily dependent 
on frontal lobe integrity. We hypothesized that aspects of executive function would be impaired in the patient group.

\section{Materials and methods}

\subsection{Participants}

Inclusion criteria were a confirmed mutation of the CHRNA4 (S248F) gene, English as the first language, and absence of other neurological or cognitive disorders. Clinical features of the nine eligible participants from a single large family $[14,18]$ are summarized in Table 1 . At the current assessment, a detailed clinical history and neurological examination were obtained by M.F. and I.E.S., and the participants' medical records were reviewed and the most recent carbamazepine serum level (usually within 2 days of testing) was obtained. Data recorded included age at seizure onset and offset, weekly seizure frequency estimated over the duration of the illness and at the time of testing, antiepileptic medication at the time of assessment and most recent drug levels, and smoking status. Two investigators (I.E.S., S.F.B.) reviewed the medical details and rated the epilepsy as mild or severe. This rating was used as a measure of the impact of the disease at the time patients had active seizures. Patients who were seizure free with or without the use of medication were categorized as having mild epilepsy; those with ongoing seizures despite medication or a history of greater than 15 years of seizures were regarded as having severe epilepsy. At the time of assessment all but one participant with ADNFLE was seizure free (see Table 1), and six participants with ADNFLE took antiepileptic medication (carbamazepine in 6 , additional clonazepam in 1 ). The average age at seizure onset was 7.2 years (range: $5-12$ ), and seizures occurred for an average of 23.2 years (range: $2-54$ ). The average number of seizures per week during that time was 10.3 (range: 5-40).

To control for intrafamily effects independent of the mutation we calculated the degree of relatedness, a coefficient of the proportion of genetic identity shared between members of the pedigree and a proband. For example, $50 \%$ of the proband's genome is shared by siblings and parents, yielding a relatedness coefficient of 0.5. A group of age-, sex-, and education-matched control participants were recruited. There were two males in each group. All participants provided written informed consent, in accordance with institutional and Australian National Health and Medical Research Council guidelines. Ethical approval was provided by the Austin Health Human Research Ethics Committee.

\subsection{Cognitive measures}

Verbal fluency was measured using the standard clinical administration of the Controlled Oral Word Association Test (COWAT) (see Strauss et al. [19]). The cognitive demands and neural substrate of this task are well described $[20,21]$. The measure used here was the total number of words retrieved for the letters F, A, and $\mathrm{S}$.

To examine in detail aspects of frontal lobe function, we developed measures using in-house programming (either EPrime or matlab), and all tasks were presented on a laptop computer. The ability to inhibit a prepotent response was measured using Color-Word Interference (Stroop task). The task comprised 120 trials in which a single word was presented on the screen. Incongruent trials (i.e., where the color word and the font's color did not match) constituted $30 \%$ of the trials. Participants named the font color as quickly as possible, and accuracy and reaction time were measured using software developed in matlab (Version 6, The Mathworks).

Vigilance (sustained attention) was measured by assessing accuracy of performance over 15 minutes while participants pressed a button in response to single letters presented on the laptop screen. Two trial types were recorded. Target trials required participants to press one key each time a letter was seen on the screen. "Change" trials occurred infrequently (18\%) and required an alternative button press when the letter that was presented was ' $c$ ', and it was preceded by the letter ' $x$ ', and they were in different colors. Thus, the task required monitoring of the $n-1$ item's features (akin to $n$-back working memory), inhibition of a prepotent motor response, which, although slightly modified, resembles a go/no go task, as well as vigilance. Accuracy was measured across the length of the task and within four equal time epochs.

Delayed match-to-sample is a task requiring participants to select a previously presented target stimulus from a group of distractors. Here we used a short delay of 2 seconds. In our task, a complex pictorial target was shown on a screen and, 2 seconds later, was presented again with three "foil" stimuli that shared either color or pattern features. The short delay period meant that this task relied more heavily on immediate attention rather than memory processing.

Attentional set shifting during a rule learning task was assessed using a paradigm based on intradimensional and extradimensional set shifting [22]. Participants viewed a computer screen on which three boxes were presented, from left to right across the screen. On each trial, two of three boxes on the screen contained a stimulus comprising three features (animal, shape, color). Participants were instructed that there was a rule they needed to learn, based on those features, and that after each trial they would be provided feedback to assist their learning. Responses were made using one of three sequential buttons on the keyboard, which corresponded to the position of the boxes on the screen. After each response, feedback (correct/incorrect) was provided visually on the screen and the trial was repeated if incorrect. After eight correct responses, the rule changed and the participant was required to

Table 1

Clinical and demographic characteristics of participants with the mutation.

\begin{tabular}{|c|c|c|c|c|c|c|}
\hline Pedigree reference ${ }^{\mathrm{a}}$ & Age at assessment & Age at onset (years) & Duration (years) & Seizure frequency (weekly) ${ }^{\mathrm{b}}$ & AEDs at time of interview & Disease severity \\
\hline IV-22 & 62 & 7 & 48 & 5 & $\mathrm{CBZ}^{\mathrm{C}}$ & Severe \\
\hline IV-27 & 63 & 6 & 54 & 5 & $\mathrm{CBZ}$ & Severe \\
\hline V-3 & 43 & 7 & 34 & 5 & $\mathrm{CBZ}+\mathrm{CLN}$ & Severe \\
\hline $\mathrm{V}-4$ & 41 & 5 & 10 & 5 & - & Mild \\
\hline$V-12^{d}$ & 37 & 6 & 31 & 40 & $\mathrm{CBZ}$ & Severe \\
\hline V-15 & 33 & 5 & 12 & 12 & $\mathrm{CBZ}$ & Mild \\
\hline $\mathrm{V}-28$ & 38 & 6 & 12 & 10 & - & Mild \\
\hline V-33 & 28 & 12 & 6 & 1 & - & Mild \\
\hline VI-7 $7^{e}$ & 16 & 11 & 2 & 10 & $\mathrm{CBZ}$ & Mild \\
\hline
\end{tabular}

a Pedigree references refer to Fig. 3 in Steinlein et al. [3].

b Seizure frequency was obtained through medical interview.

c CBZ, carbamazepine; CLN, clonazepam.

d Ongoing seizures at the time of assessment.

e VI-7 mutation status was confirmed subsequent to earlier publications. 
adapt to the new rule requirements. Measures included the total number of errors made, total number of trials to successfully negotiate the overall task, as well as number of errors and number of trials to criterion for each rule learned.

One of the rules within the set shifting task was akin to object alternation, where the correct choice from two displayed items changed from trial to trial. This task is typically employed in reward-based learning paradigms in human and nonhuman primate literature. In our task, the participant was required not only to detect the feature (animal, shape, or color) on which the rule was based, but also to determine that this changed between two of the three options. The number of incorrect responses and the total number of trials to criterion (eight correct in sequence) were recorded.

To exclude the possibility that performance on these very sensitive tasks reflected group differences in general intellectual abilities, we used the four-subtest version of the Wechsler Abbreviated Scale of Intelligence (WASI) [23] as a measure of Full Scale IQ. Furthermore, we sought to establish whether the group with ADNFLE exhibited a frontal lobe-type syndrome through clinical interview and quantitative screening of neurobehavioral features using the Frontal Assessment Battery [24]. Participants with ADNFLE were also assessed on standard tasks of memory including the Rey Auditory Verbal Learning Test (RAVLT), and visual memory was assessed using Rey's Complex Figure (30-minute delay protocol; see Lezak et al. [25]). $z$ score statistics were computed for these tasks; RAVLT normative data were derived from Geffen et al. [26], and for Rey's Complex Figure we used data from Spreen and Strauss [27]. Audioverbal attention (Digit Span from Wechsler Adult Intelligence Scale, Third Edition (WAIS-3)) [28] and working memory (Letter Number Sequencing from the WAIS-3) were also assessed in the patient group. The Trail Making Test is a widely used clinical task that provides a measure of basic psychomotor speed (Trails A) and assesses mental set shifting abilities (Trails B). Normative data cited in Spreen and Strauss [27] were used to establish the performance of patients with ADNFLE.

\subsection{Statistical analysis}

Independent $t$ tests were used to compare the patient and control groups on cognitive measures (using a criterion of $P<0.05$, two-tailed). For any cognitive task showing a difference between the groups, we then examined performance by the patients on that task in relation to epilepsy variables. For continuous variables (age at seizure onset, duration of epilepsy, weekly seizure frequency, drug levels), we used correlation analysis. To identify and rule out cognitive variables with even a trend toward being influenced by disease variables, we used a threshold of $P=0.1$. For dichotomous variables (medication status, seizure severity, smoking status) independent $t$ tests were used with a threshold of $P=0.1$. This conservative approach was taken to carefully exclude the possibility that the differences between the groups were due to disease factors.

\section{Results}

The nine patients and nine controls were well matched on age and general intellectual abilities (all $P>0.05$ ). The mean age of the mutation group was 40.1 years $(S D=15.0$, range $=16-63$ ), and that for controls was 39.3 years $(S D=14.6$, range $=15-64)$.

\subsection{Performance by patients on standard clinical neuropsychological measures}

Full Scale IQ fell within the "average" range for both groups (mutation group: $94.0 \pm 11.8$, mean $\pm \mathrm{SD}$; control group:
$97.7 \pm 9.5$ ). The average score of patients with ADNFLE on the Frontal Assessment Battery $(15.4 \pm 1.5)$ was within normal limits. Performance on standard clinical tasks was evaluated against established normative data. Participants with ADNFLE performed lower than average on RAVLT total recall ( $z$ score, Trials $1-5:-1.4 \pm 1.0$ ) and post-interference recall (Trial 6: $-1.4 \pm 1.4$ ). Performance on the RAVLT was related to the serum level of carbamazepine (RAVLT Trial 6 scores: $r=-0.8, P=0.01$; RAVLT total scores: $r=-0.8, P=0.02$ ). Digit Span scaled scores were, on average, $6.8 \pm 2.2$. Letter Number Sequencing scaled scores $(7.0 \pm 2.9)$ were significantly better in those with "mild" disease versus those whose epilepsy was classified as "severe" $(t[6]=2.6, P=0.04)$. Delayed recall of Rey's Complex Figure was within normal limits across the group $(z$ score $=-0.3 \pm 1.2)$. Performance on Part B of the Trail Making Test, which measures set shifting using a psychomotor component, in those with the mutation was 1.4 SD above the population mean, indicating that performance was slower than expected. Performance on this variable was not related to any disease variable. Trails A performance was within normal limits $($ mean $=0.65, \mathrm{SD}=1.25$ ).

\subsection{Performance by the groups on frontal lobe measures}

Performance on several tasks differed significantly between the groups. Here we present data on those measures where no association between disease variables and performance by the patient group was identified. We adopted this approach to ensure that group differences represented ADNFLE-specific findings rather than effects due to the treatment or severity of epilepsy per se. Patients with ADNFLE performed more poorly on the object alternation phase of the set-shifting paradigm, using almost twice as many attempts to learn the rule to criterion than controls (Fig. 1) $(t[16]=-2.12, P=0.05)$. Performance was characterized by variability in rule learning, reflected by an almost threefold increase in error rate $(t[16]=-2.33, P=0.03)$. The pattern of results reflects the participants' difficulty in modifying their erroneous responses to this complex and cognitively challenging task, which relies on the ability to shift and factor in feedback on a trial-by-trial basis. This was also consistent with the formal evaluation of response inhibition using our computerized version of the Stroop test. Significantly more errors on incongruent trials of the Stroop colornaming task were seen in those with the mutation $(t[16]=3.93$, $P=0.001)$, despite the two groups' similar levels of accuracy on congruent trials $(t[16]=1.71, P=0.11)$ (Fig. 2$)$. Verbal fluency performance fell within the average range for control participants, but

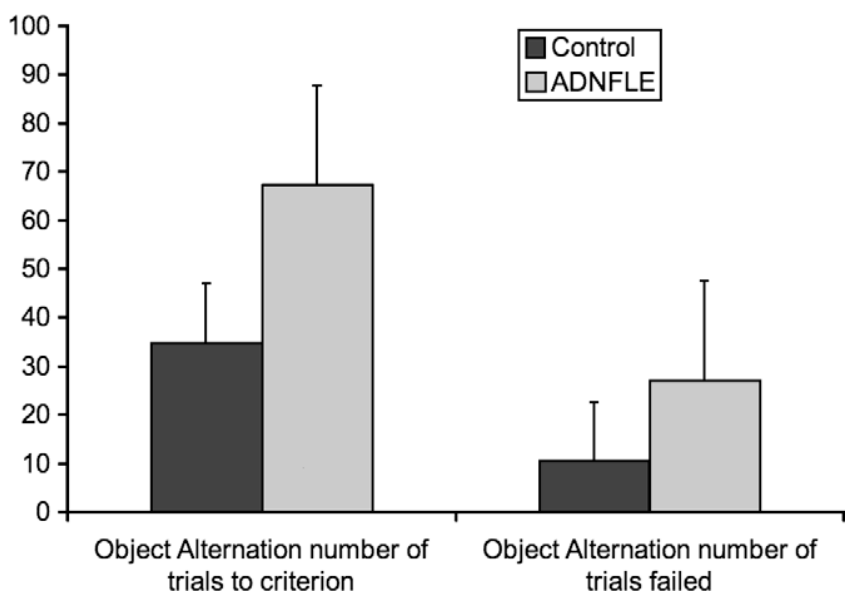

Fig. 1. Those with the CHRNA4 S248F mutation performed significantly more poorly on the object alternation task, with a threefold increase in the average number of attempts (error bars represent SE) required to learn the rule and a concomitant increase in the number of errors made during the task. 


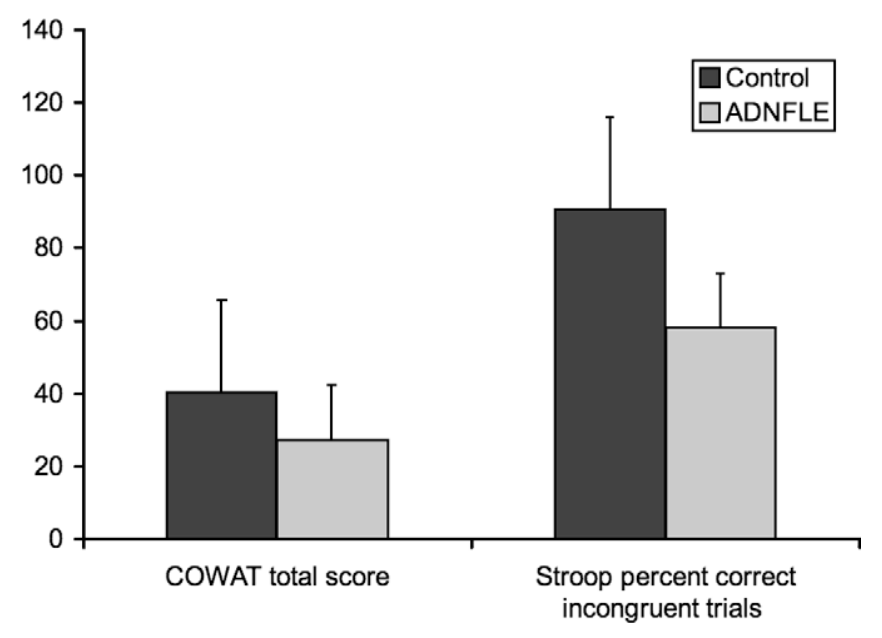

Fig. 2. Performance on COWAT was in the average range for controls, but significantly worse in patients with the CHRNA4 S248F mutation. The proportion of correct incongruent trials on the Stroop Color-Word interference task was also significantly worse in those with the mutation than in controls. Scores are mean values and error bars represent SE.

was significantly worse for those with the mutation $(t[16]=2.9$, $P=0.01$ ). The number of trials performed correctly on the delayed matching-to-sample task was similar for the two groups.

The measure of vigilance that we employed comprised a continuous performance element (measuring sustained attention) as well as a more demanding requirement to change response set at random, infrequent time points throughout (akin to a go/no go paradigm). Task performance can be collapsed across the entire 15 minutes or divided into time epochs of equal duration. The latter approach permits a closer analysis of change in attention over time. First, examining the overall performance across all trials showed that performance on the sustained attention component differed between the groups (e.g., total percentage correct: $t[15]=3.7$, $P=0.002$ ), but, in patients with ADNFLE, was significantly worse in patients whose epilepsy was rated as severe $(t[6]=2.1, P=0.08)$ and in those still on medication $(t[6]=-2.7, P=0.03)$. A significant correlation with serum level of medication and total percentage correct was also identified $(r=-0.7, P=0.05)$. Trials on which participants had to change their responses based on the sequence of presented items demanded higher order cognitive skills. The total percentage correct on all such "change" trials did not differ between patients with ADNFLE and matched controls ("change" trials: $t[15]=0.97, P=0.35$ ), and repeated-measures analysis of the four epochs did not reach statistical significance $(F[3.45]=2.4$, $P=0.07)$.

\section{Discussion}

In contrast to previous research in patients with ADNFLE, our study involves a single group, which was homogeneous with respect to the underlying causative mutation. Family members with the S248F CHRNA4 subunit mutation were impaired on tasks that require flexible adaptation to, and monitoring of, cognitive demands. In particular, performance was impaired on Color-Word Interference, object alternation, Controlled Oral Word Association Test, and Part B of the Trail Making Test, implicating a frontal lobe dysfunction. General intellectual function was normal, suggesting that the cognitive difficulties identified cannot be attributed to a global impairment. Like others, we identified memory difficulties in this cohort, but showed that these relate to the severity of disease-related variables or medications.

Performance on the frontal lobe tasks in the patient group was independent of seizure severity, medication, or smoking. An intra- familial genetic effect, other than the mutation of interest, seems unlikely as defects did not depend on the degree of relatedness in this large pedigree [14]. A more definitive evaluation of this question could be achieved by studying mutation-negative family members, but this was not possible in the current study. This is an interesting area for future research. The cognitive profile in our patients is similar to that seen in lesional frontal lobe epilepsy [29]. In our patient group, however, there is no detectable lesion on MRI. On balance, the lack of association between impaired cognitive flexibility and familial factors, seizure effects, epilepsy treatment, or nicotine exposure increases the likelihood of a gene-specific effect. Converging evidence could be obtained in future studies by investigating the effects of nAChR agonists or antagonists on cognitive flexibility in patients with the mutation.

In previous research on CHRNA4 mutations, visuospatial attention "scaling" was better in those with a greater number of $C$ alleles in the CHRNA4 C1545T single-nucleotide polymorphism (SNP) $[30,31]$. Visuospatial attention scaling refers to the modification of an attentional focus in the visual field on the basis of prior information. The authors describe it as "a flexibly deployed gradient that can be dynamically constricted or expanded ... according to task demands" ([30], p. 3.) This paradigm bears a class relationship to the tasks that we used, lending weight to our hypothesis that core executive functions are supported by the $\alpha 4$ receptor.

In accord with our study, executive dysfunction has also been demonstrated in four families with $\alpha 4$ subunit mutations, S252L and T265I, as well as S248F [16]. Like the current study, verbal learning impairments have been identified previously in patients with ADNFLE. This included patients with S248F mutation [16] and twins with a novel mutation of the $\beta 2 \mathrm{nAChR}$ [17]. The latter had significant impairments of general intellectual skills and other aspects of verbal functions. Further, a mother and daughter with a CHRNB2 mutation and relatively intact intellectual skills [32] showed memory impairments, as well as set shifting difficulties. In the group studied here, verbal learning, audioverbal span, and working memory performances were correlated with disease and/or treatment variables, preventing, or rendering less likely, a direct causal link between these measures and mutations in ADNFLE.

Since our original description of ADNFLE, greater variability in the general intellectual profile of patients with nAChR subunit mutations has been recognized. This variability may be family, and thus presumably nAChR gene mutation, dependent. Cho and colleagues described a different family with ADNFLE (CHRNA4 mutation) and markedly impaired general intellectual skills [33]. Similarly, we found that severe ADNFLE can be associated with marked psychiatric disability and intellectual impairment [34]. The molecular mechanisms underlying different intellectual outcomes are not yet understood. The current study also found that the group performed within the normal range on the Frontal Assessment Battery. This bedside screening measure was designed for the detection of frontal lobe syndrome arising from destructive frontal lobe disease (injury, hemorrhages, degeneration, etc.). Our patients do not present with a clinically evident frontal lobe syndrome; hence our use of sophisticated high-level cognitive paradigms to illustrate the effects of receptor level dysfunction in ADNFLE.

There is now an emerging literature describing cognitive abilities in patients with ADNFLE and different mutations. The cognitive phenotype appears to be variable, although the association between cognitive performance and epilepsy-related variables has not always been examined. Although previous authors have speculated about links between mutation and specific cognitive domains [16], we agree that it is premature to draw such conclusions. Indeed, before concluding that there may be a specific phenotype associated with ADNFLE, it is important to first examine secondary factors that 
may explain cognitive findings. Although we cannot rule out the possibility that disease variables may, at least in part, explain our results, an important focus of our study was the careful examination of available disease variables in relation to task performance. A characteristic of ADNFLE is frequent, brief, nocturnal seizures that disturb normal sleep. Only one of our participants with the mutation continued to have seizures at the time of assessment, minimizing any effects of chronic sleep loss on our findings. The focal nature of the impairment in patients with $\alpha 4$ mutations is consistent with the in vivo finding of reduced prefrontal nAChR binding [35], and contrasts with the widespread physiological distribution of $\alpha 4 \beta 2$ nAChRs [5]. Accordingly, we conclude that cognitive flexibility is a core component of the neuropsychological phenotype associated with mutations causing ADNFLE. Additional research examining the impact of the gene on the developing brain and cognitive skill acquisition is warranted.

\section{Ethical approval}

We confirm that we have read the Journal's position on issues involved in ethical publication and affirm that this report is consistent with those guidelines.

\section{Conflict of interest statement}

None of the authors has any conflict of interest to disclose.

\section{Acknowledgments}

The contributions of family members, control participants, and Ms. Danya Vears are gratefully acknowledged. This work was supported by National Health and Medical Research Council of Australia program funding to S.F.B., I.E.S., and D.C.R. and postdoctoral training fellowship funding (ID 251755) to A.G.W.

\section{References}

[1] Aridon P, Marini C, Di Resta C, et al. Increased sensitivity of the neuronal nicotinic receptor $\alpha 2$ subunit causes familial epilepsy with nocturnal wandering and ictal fear. Am J Hum Genet 2006;79:342-50.

[2] De Fusco M, Becchetti A, Patrignani A. The nicotinic receptor B2 subunit is mutant in nocturnal frontal lobe epilepsy. Nat Genet 2000;26:275-6.

[3] Steinlein O, Mulley JC, Propping P. A missense mutation in the neuronal nicotinic acetylcholine receptor alpha 4 subunit is associated with autosomal dominant nocturnal frontal lobe epilepsy. Nat Genet 1995;11(2):201-3.

[4] Heishman SJ, Taylor RC, Hennigfiels JE. Nicotine and smoking: a review of effects on human performance. Exp Clin Psychopharmacol 1994;2:1-51.

[5] Levin ED. Nicotinic receptor subtypes and cognitive function. J Neurobiol 2002;53:633-40.

[6] Levin ED, Connors CK, Silva D. Transdermal nicotine effects on attention. Psychopharmacology 1998;138:217-30.

[7] Levin ED, Connors CK, Sparrow E. Nicotine effects on adults with attentiondeficit/hyperactivity disorder. Psychopharmacology 1996;123:55-63.

[8] Mancuso G, Warburton DM, Melen M, Sherwood N, Tirelli E. Selective effects of nicotine on attentional processes. Psychopharmacology 1999;146:199-204.
[9] Min SK, Moon IW, Ko RW, Shin HS. Effects of transdermal nicotine on attention and memory in healthy elderly non-smokers. Psychopharmacology 2001;159:83-8.

[10] Brown RW, Beale KS. Mecamylamine blocks enhancement of reference memory but not working memory produced by post-training injection of nicotine in rats tested on the radial arm maze. Behav Brain Res 2002;134:259-65.

[11] Griesar WS, Zajdel DP, Oken BS. Nicotine effects on alertness and spatial attention in non-smokers. Nicotine Tobacco Res 2002;4:185-94.

[12] Newhouse PA, Sunderland T, Tariot PN. Intravenous nicotine in Alzheimer's disease: a pilot study. Psychopharmacology 1988;95:171-5

[13] Rezvani AH, Bushell PJ, Levin ED. Effects of nicotine and mecamylamine on choice accuracy in an operant visual signal detection task in female rats. Psychopharmacology 2002;164:369-75.

[14] Scheffer I, Bhatia K, Lopes-Cendes I. Autosomal dominant nocturnal frontal lobe epilepsy: a distinctive clinical disorder. Brain 1995;118:61-73.

[15] Steinlein O, Bertrand D. Neuronal nicotinic acetylcholine receptors: from the genetic analysis to neurological diseases. Biochem Pharmacol 2008;76:1175-83.

[16] Picard F, Pegna A, Arntsberg V. Neuropsychological disturbances in frontal lobe epilepsy due to mutated nicotinic receptors. Epilepsy Behav 2009;14:354-9.

[17] Bertrand D, Elmslie F, Hughes E. The CHRNB2 mutation I312M is associated with epilepsy and distinct memory deficits. Neurobiol Dis 2005;20:799-804.

[18] Scheffer I, Bhatia K, Lopes-Cendes I. Autosomal dominant frontal lobe epilepsy misdiagnosed as sleep disorder. Lancet 1994;343:515-7.

[19] Strauss E, Sherman EMS, Spreen O. A compendium of neuropsychological tests: administration, norms, and commentary. 3rd ed. New York: Oxford University Press; 2006.

[20] Stuss DT, Alexander MP, Hamer L. The effects of focal anterior and posterior brain lesions on verbal fluency. J Int Neuropsychol Soc 1998;4:265-78.

[21] Wood AG, Saling MM, Abbott DF, Jackson GD. A neurocognitive account of frontal lobe involvement in orthographic lexical retrieval: an fMRI study. NeuroImage 2001;14:162-9.

[22] Beats B, Sahakian B, Levy R. Cognitive performance in tests sensitive to frontal lobe dysfunction in the elderly depressed. Psychol Med 1996;26:591-603.

[23] Wechsler D. Wechsler Abbreviated Scale of Intelligence (WASI). San Antonio, TX: Harcourt Assessment; 1999.

[24] Dubois B, Slachevsky A, Litvan I, Pillon B. The FAB: a frontal assessment battery at bedside. Neurology 2000;55:1621-6.

[25] Lezak MD, Howieson DB, Loring DW. Neuropsychological assessment. 4th ed. Oxford: Oxford University Press; 2004.

[26] Geffen G, Moar KJ, O'Hanlon AP, Clark CR, Geffen LB. Performance measures of 16- to 86-year-old males and females on the Auditory Verbal Learning Test. The Clin Neuropsychol 1990;4:45-63.

[27] Spreen O, Strauss E. A compendium of neuropsychological tests: administration, norms, and commentary. 2nd ed. New York: Oxford University Press; 1998

[28] Wechsler D. Wechsler Adult Intelligence Scale-third edition (WAIS-3). San Antonio, TX: Harcourt Assessment; 1997.

[29] Helmstaedter C. Behavioral aspects of frontal lobe epilepsy. Epilepsy Behav 2001;2:384-95.

[30] Greenwood PM, Fossella JA, Parasuraman R. Specificity of the effect of a nicotinic receptor polymorphism on individual differences in visuospatial attention. J Cogn Neurosci 2005;17:1611-20.

[31] Parasuraman R, Greenwood PM, Kumar R, Fossella JA. Beyond heritability: neurotransmitter genes differentially modulate visuospatial attention and working memory. Psychol Sci 2005;16:200-7.

[32] Cho Y-W, Yi S-D, Lim J-G, Kim J-G, Motamedi GK. Autosomal dominant nocturnal frontal lobe epilepsy and mild memory impairment associated with CHRNB2 mutation I312M in the neuronal nicotinic acetylcholine receptor. Epilepsy Behav 2008;13:361-5.

[33] Cho YW, Motamedi GK, Laufenberg I. A Korean kindred with autosomal dominant nocturnal frontal lobe epilepsy and mental retardation. Arch Neurol 2003;60:1625-32.

[34] Derry CP, Heron SE, Phillips F. Severe autosomal dominant nocturnal frontal lobe epilepsy associated with psychiatric disorders and intellectual disability. Epilepsia 2008;49:2125-9.

[35] Picard F, Bruel D, Servent D. Alteration of the in vivo nicotinic receptor density in ADNFLE patients: a PET study. Brain 2006;129:2047-60. 